\title{
PULMONARY VASCULAR SCLEROSIS WITH RIGHT VENTRICULAR FAILURE
}

\author{
BY \\ A. M. BARRETT AND LESLIE COLE \\ From Addenbrooke's Hospital and the Department of Pathology, University of Cambridge \\ Received December 19, 1945
}

The following is an account of a patient with pulmonary vascular sclerosis who died of right ventricular failure within four months of the appearance of the first symptom of ill health.

A navvy charge hand, aged 34, was admitted to Addenbrooke's Hospital on November 6, 1942. In August 1942 he noticed that some of his teeth were very loose and tended to bleed easily, and shortly after this that he was breathless on exertion. Until then he had always been fit with no cough or breathlessness. In early October he went to the dentist, and twelve teeth were extracted under general anæsthesia. Following these extractions breathlessness on exertion gradually increased so that when he was admitted to hospital he could walk up six steps only with difficulty. A week before admission he had also noticed slight swelling of his left leg, occasional palpitation, and some upper abdominal pain and flatulence after food. His appetite remained good and his weight constant. His work had not been physically very strenuous but his hours had been long-from eight in the morning sometimes until midnight. As this was seven miles from home he used to cycle to and fro. He often worked a seven-day week.

In the past he had had no serious illnesses, no rheumatism, nor rheumatic fever, nor other condition that might predispose to cardiac trouble. He had been accustomed to smoke up to 50 cigarettes a day and drank a moderate amount of beer but no spirits. His family history was healthy and did not suggest any tendency to arteriosclerosis or cardiac disease. His mother and father and brothers and sisters were all alive and well.

On admission he was very breathless and slightly cyanosed. The pulse was regular-rate 100 . Blood pressure $140 / 90$, respiratory rate 20 , temperature normal. A teleradiogram showed that the healt was enlarged $(15.3 \mathrm{~cm}$. in the transverse diameter) with marked bulging of the pulmonary artery (Fig. 1). The cardiogram showed right axis deviation with inversion of T II and T III (Fig. 2). The heart sounds were poor in quality with gallop rhythm and the pulmonary second sound was accentuated. There were no murmurs, clubbing of the fingers, venous pulsation, nor edema, and no evidence of arteriosclerosis in the radial, brachial, or retinal arteries. The respiratory movements were good and there were no signs of chronic bronchitis or emphysema; a few crepitations were audible at the right base.

The liver appeared congested, the edge being palpable two inches below the costal margin. There was no abdominal tenderness and no evidence of ascites. The remaining teeth appeared healthy and the gums had healed well following extraction. The urine contained a trace of albumin, but was otherwise normal.

Blood count showed 5.6 million red corpuscles with hæmoglobin 112 per cent and 12,800 leucocytes with a normal differential count. Blood culture was sterile and the Wasserman reaction of the blood was negative. Blood urea $37 \mathrm{mg}$. per 100 c.c. Blood sedimentation rate on two occasions 1 and $4 \mathrm{~mm}$. per hour respectively.

Progress. In spite of treatment by rest and full digitilization, progress was steadily downhill from the day of admission until death on December 2, 1942. During this period there was practically no change in either the pulse rate or the respiratory rate. Breathlessness and distress on the slightest exertion became progressively more marked and cyanosis steadily increased.

\section{POST-MORTEM EXAMINATION}

Congestion of the lungs, the lower lobes being tough and slightly nodular in consistency and the cut surface of the anterior borders yellowish-grey in colour, with many small rusty-red patches. Considerable emphysema of anterior borders only, without noticeable distention of the lung or formation of bullæ. One small fragment of ante-mortem thrombus in a small pulmonary artery in the right lower lobe, the area supplied by this vessel being hyperæmic but not infarcted. Unusually conspicuous blood vessels everywhere on the cut surfaces of the lungs. Conspicuous atheroma of main pulmonary artery and all its larger branches. Pulmonary veins normal. Only slight atheroma of aorta and its branches, almost none of coronary arteries. Slight excess (about 50 c.c.) of clear yellow fluid in 


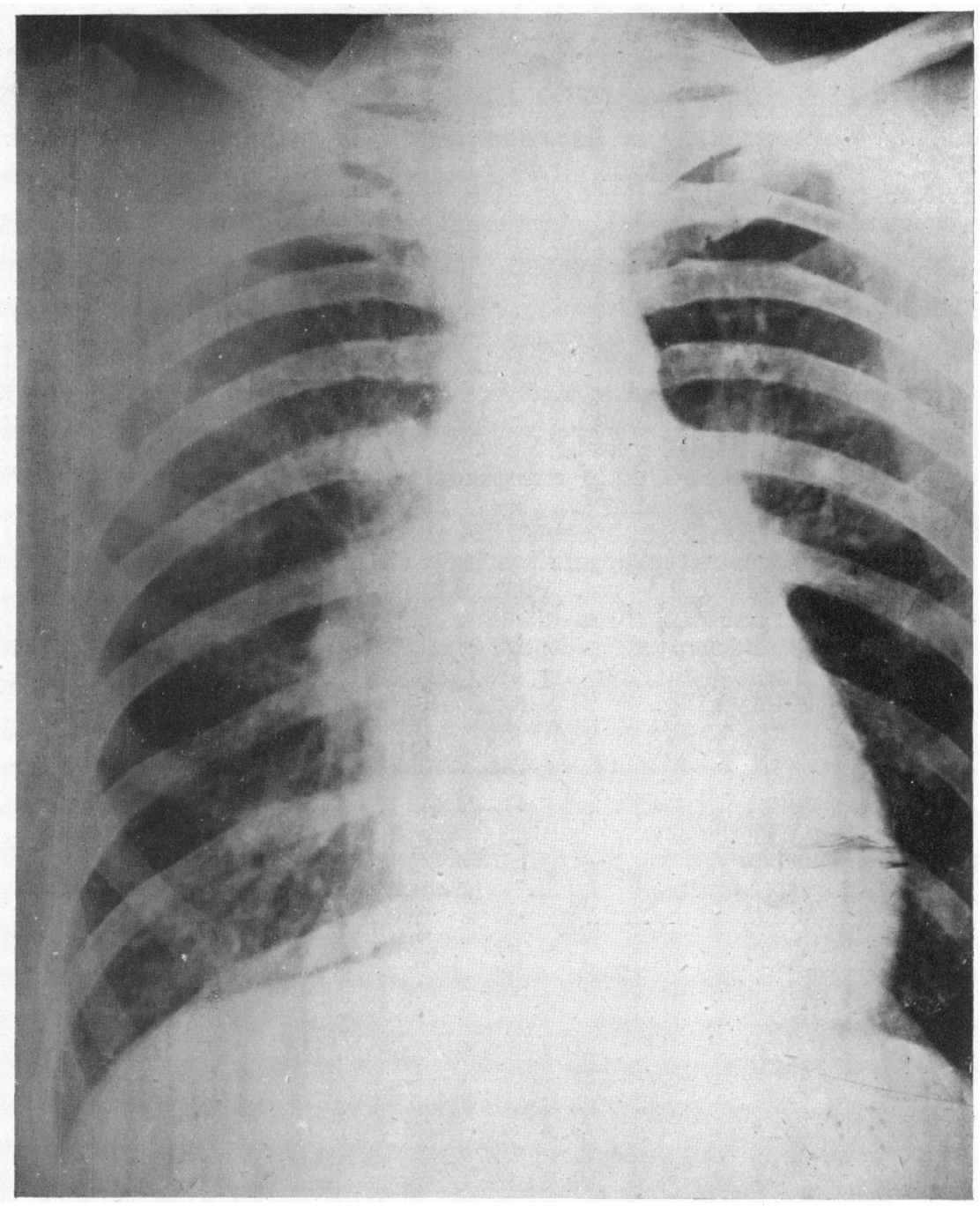

FIG. 1.-Radiogram showing large pulmonary artery.

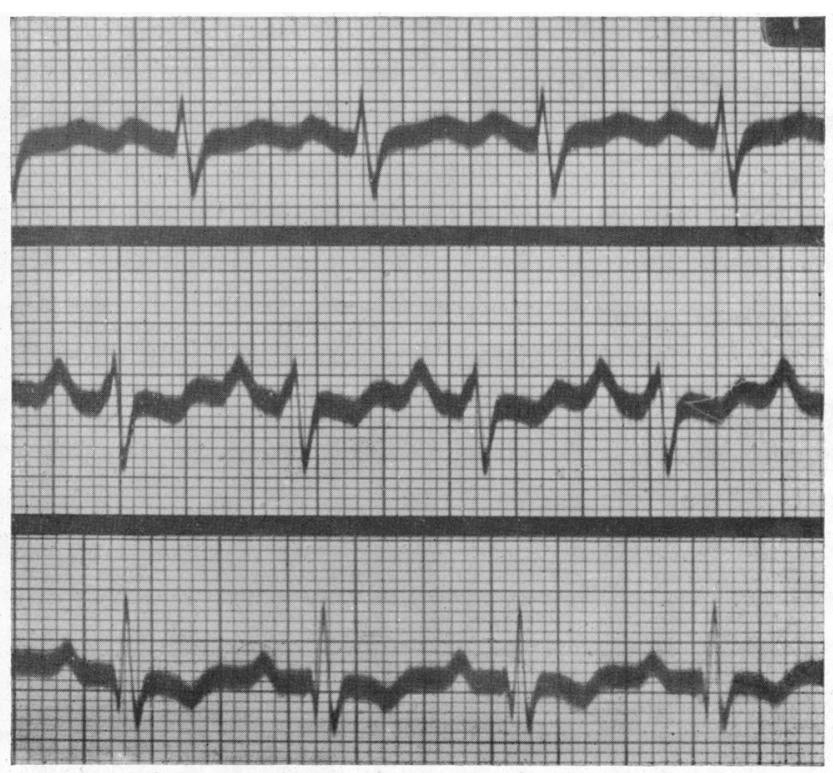

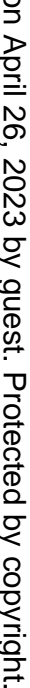

FIG. 2.-Cardiogram showing right axis deviation. 
pericardium. Great dilatation of right side of heart, the cavity of both auricle and ventricle being about double the normal size. Considerable hypertrophy of right ventricle, its wall being stiff like a piece of leather. No hypertrophy or dilatation of left auricle or ventricle. Thickness of right ventricle $0.8 \mathrm{~cm}$. Thickness of left ventricle $1.4 \mathrm{~cm}$. The degree of hypertrophy was considerably more than is represented by these figures because of the great dilatation of the right ventricle. Brownish red, rather opaque myocardium; with frequent small greyish patches $(0 \cdot 1-0 \cdot 2 \mathrm{~cm}$. diameter) in the wall of left ventricle suggesting slight fibrosis; heart valves normal. Ante-mortem thrombus in right auricular appendage and also adhering to the wall of the right auricle at the site of the foramen ovale, which was patent $(0.2 \mathrm{~cm}$. diameter) but valvular. No clot projecting through the foramen into the left auricle nor any ante-mortem.thrombus anywhere in the left side of the heart. Considerably enlarged hyperæmic and anthrocotic bronchial lymph glands, probably acutely inflamed, at hilum of left lung and at bifurcation of trachea. No abnormality of other lymph glands. Dilated stomach. Congestion and mucous catarrh of intestines. Chronic venous congestion of liver with odematous connective tissue around the normal gall-bladder.

Small effusions of clear yellow fluid in each pleural cavity and in peritoneum (about 115 c.c. in each). Moderately enlarged firm " chronic heart failure" spleen. Congestion and cloudy swelling of kidneys. Two recent infarcts and an adenoma $(0.4 \mathrm{~cm}$. diameter) in left kidney. Bladder, prostate, and urethra normal, also testes and epididymis. Suprarenals, pancreas, thyroid, and pituitary normal; thymus for the most part replaced by fat. Normal brain. Middle ears clear. Pyorrhœa around bases of remaining teeth; teeth otherwise in fair condition; gums healed at sites of absent teeth. No clubbing of fingers; no œedema. Considerable cyanosis. Well-developed, well-nourished man.

Weight of Organs. Heart 455 g., left lung 610 g., right lung 710 g., liver 2080 g., spleen 280 g., right kidney $130 \mathrm{~g}$., left kidney $155 \mathrm{~g}$.

\section{Microscopical Examinations}

The Lungs. Six sections from different parts of the lungs show extensive disintegration of the alveolar walls, although only the anterior borders of the lungs are frankly emphysematous. In some areas-usually where alveoli are intact-capillaries are greatly engorged: elsewhere the tattered alveolar walls appear almost bloodless (Fig. 3). The contrast between the sharply defined patches of capillary engorgement and the surrounding zones in which it is difficult to distinguish any capillaries

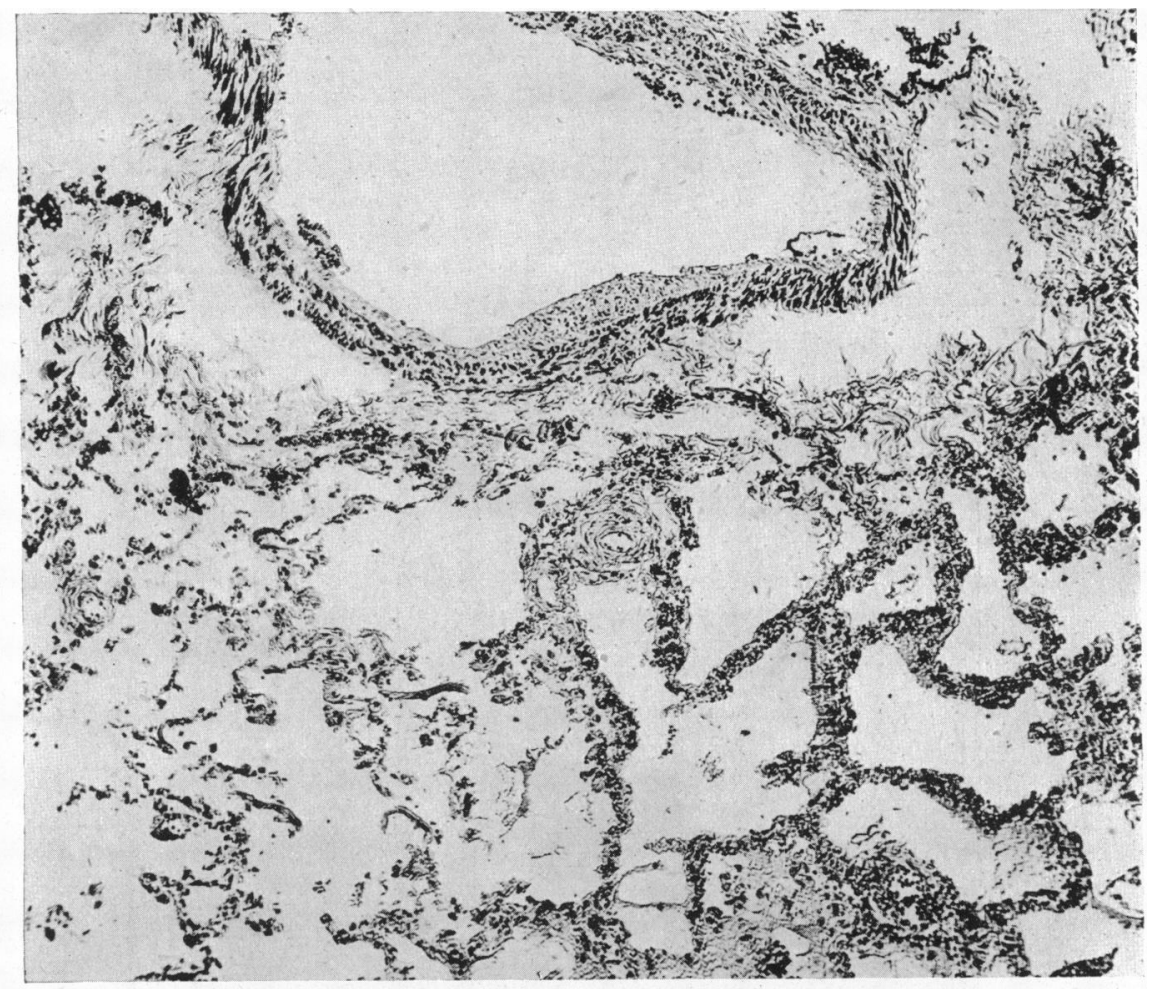

FIG. 3.-At the top, a small muscular artery with conspicuous intimal thickening; arteriole with greatly thickened wall in centre; area of capillary engorgement with preservation of alveolar pattern at bottom right; area of alveolar disintegration with few visible capillaries at bottom left.

Goldner's modification of Masson's stain. Magnification $\times 88$. 


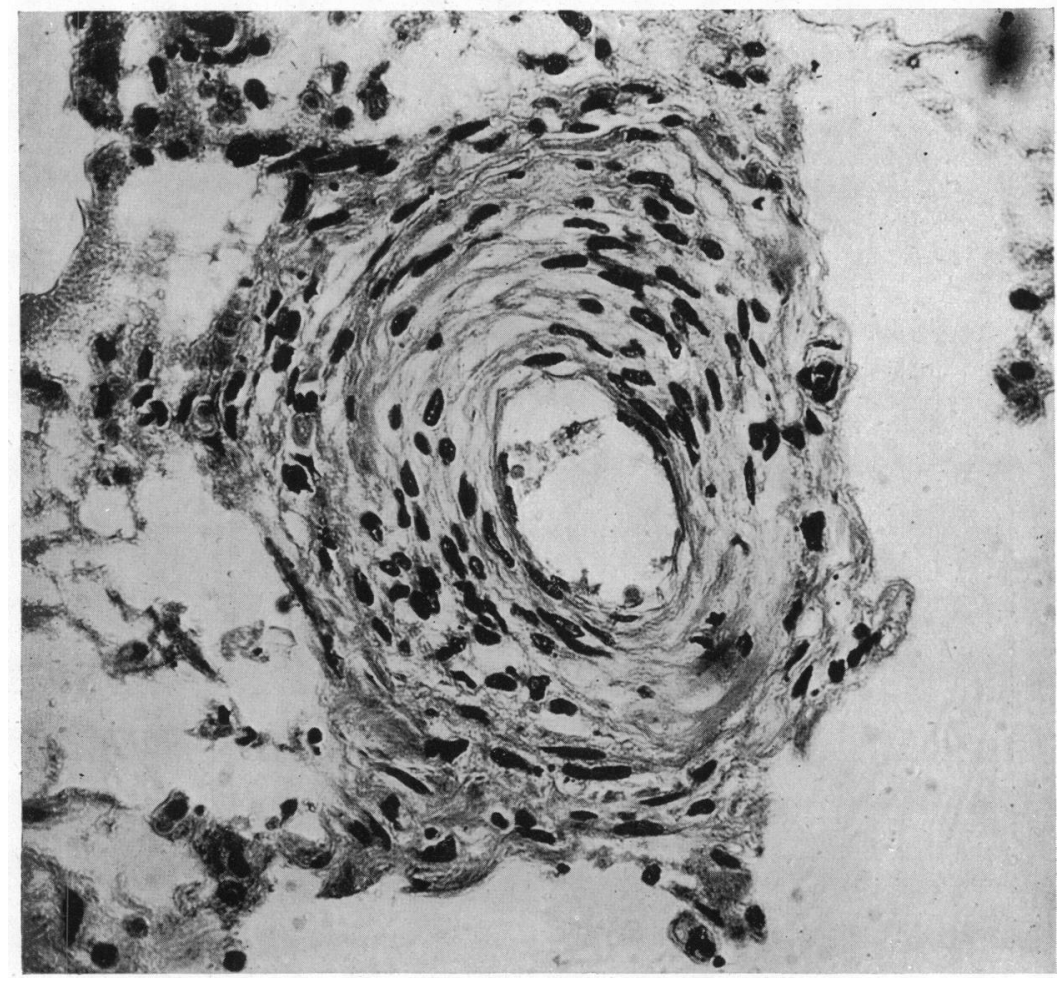

Fig. 4. -An arteriole more highly magnified, showing the characteristic lesion. Ehrlich's hæmatoxylin and eosin. Magnification $\times 390$.

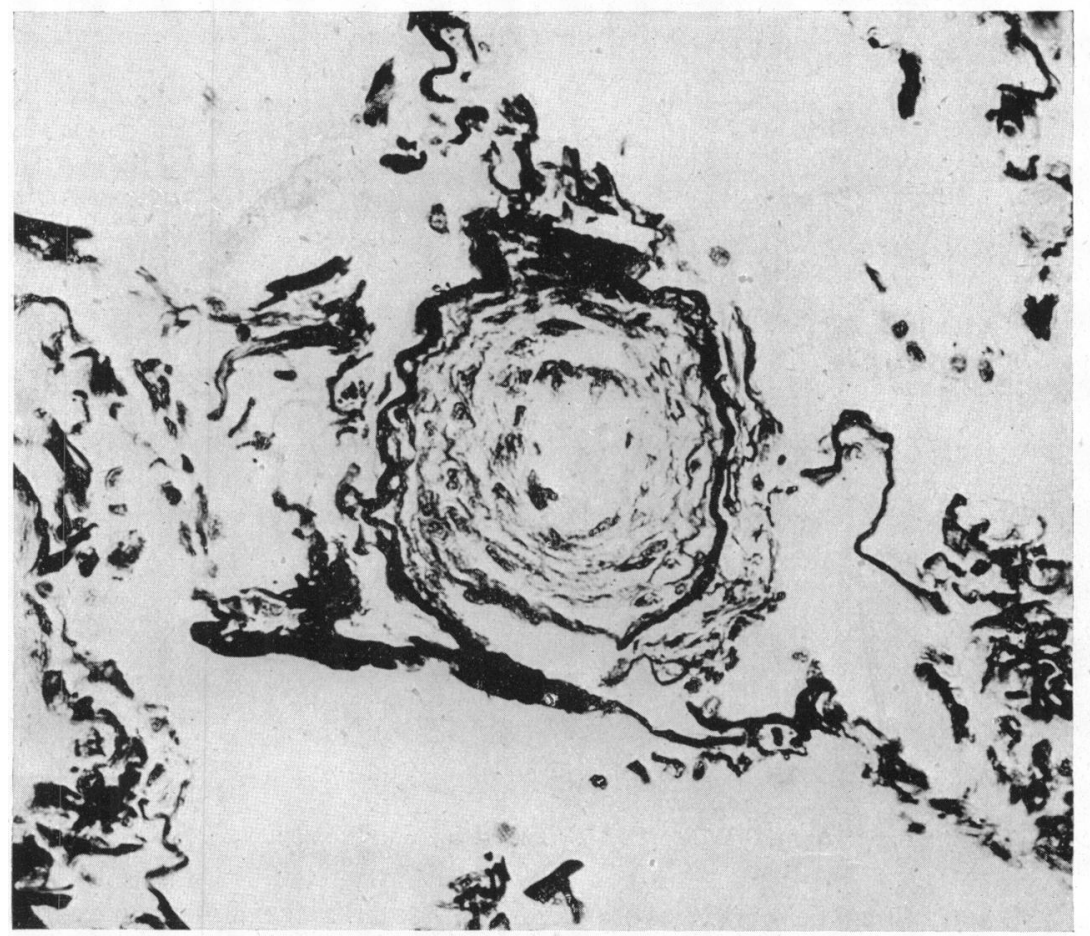

Fig. 5.-Another arteriole. The elastic fibril, which alone normally supports the endothelium, is seen as thick wavy black lines outside the concentric rings of cells.

Weigert's resorcin-fuchsin and neutral red. Magnification $\times 390$. 


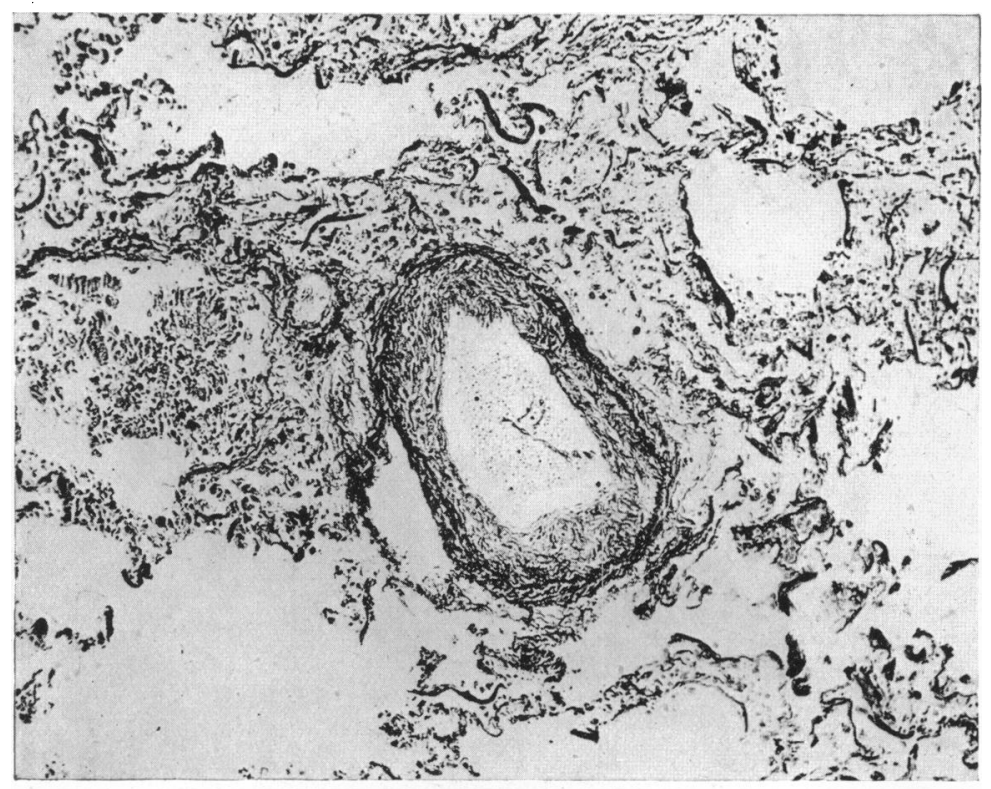

Fig. 6.-A small muscular artery. The internal and external elastic laminæ which bound the media are best seen on the left side. Weigert's resorcin-fuchsin and neutral red. Magnification $\times 88$.

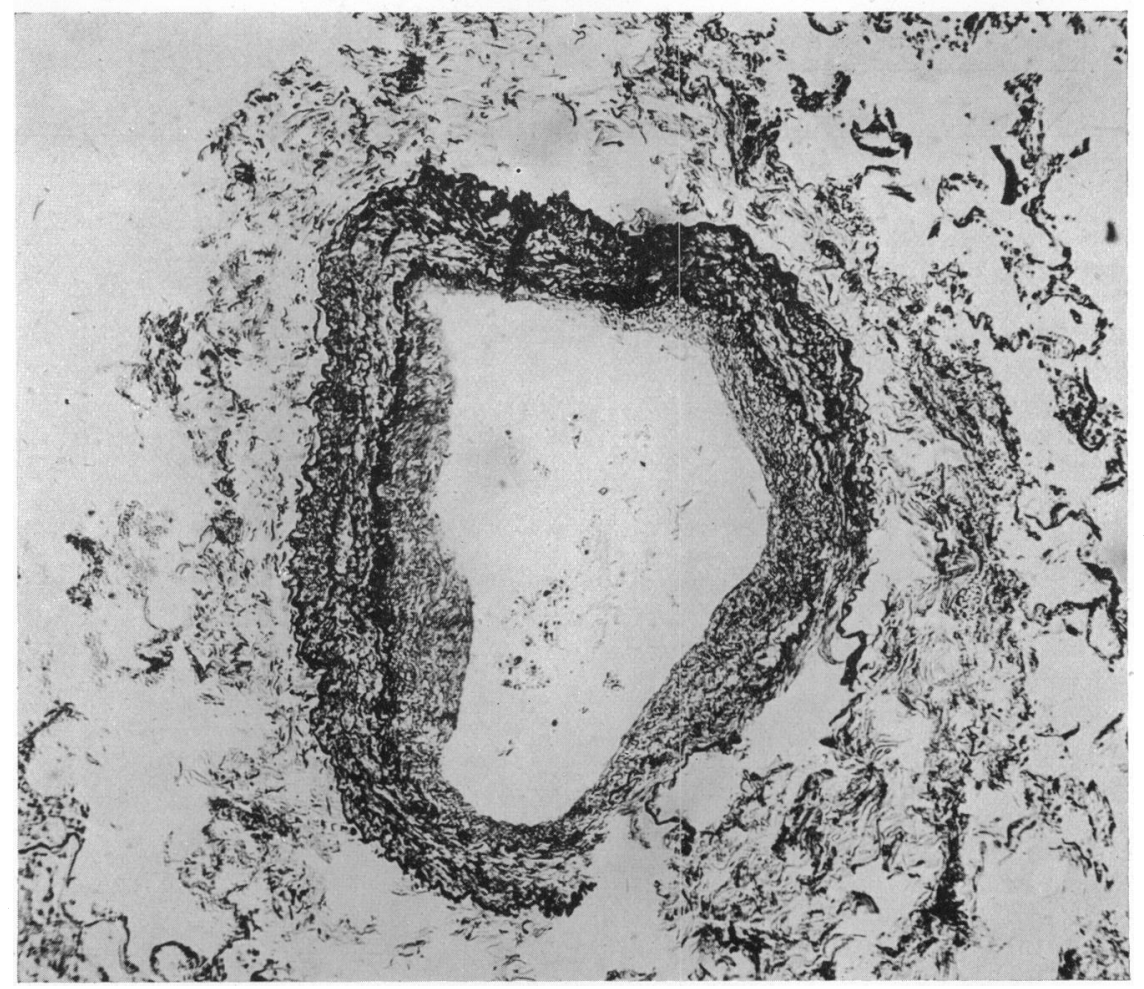

FIG. 7.-A pulmonary artery. Weigert's resorcin-fuchsin and neutral red. Magnification $\times 88$.

at all, is very striking. Small hæmorrhages are present here and there, and many macrophages contain hæmosiderin.

Conspicuous abnormalities are present in pulmonary blood vessels of all sizes but are perhaps most notable in the arterioles and smaller muscular arteries. According to Brenner (1935) the wall of the pulmonary arterioles normally consists of endothelium supported only by a spiral strand of 
elastic, but here in the affected arterioles the wall is greatly thickened and the lumen narrowed by the presence of a layer of spindle-shaped or elongated cells arranged concentrically between the endothelium and the elastic lamina (Fig. 4 and 5). The outlines of the individual cells are indistinct but in sections stained by Van Gieson's method the nuclei are set against a pale-pink background which sometimes contains a few deeper red collagen fibres. The cells are probably fibroblasts: in their appearance and staining reactions they are different from both endothelial cells and muscle fibres. Elastic fibres are not present amongst them. Sometimes their nuclei are not distributed evenly around the narrowed lumen but are arranged in two groups at opposite poles, as in the vessel shown in Fig. 6. The lesion is similar to the " hyperplastic arteriosclerosis" or " endarteritis fibrosa" of the arterioles of the kidney and other tissues in malignant hypertension; it is different from the true endarteritis obliterans of inflammation.

Great intimal thickening is also seen in many small muscular arteries in the lungs (Fig. 3 and 6). Here the thickened intima contains relatively fewer, more elongated and denser nuclei than in the arterioles, and fine elastic fibres and muscle fibres are present as well as collagen. There is no re-duplication of the internal elastic lamina. The media appears normal in structure but may be thickened, though the measurements that would be necessary to establish this have not been made.

In the larger pulmonary arteries (Fig. 7) both medial and intimal hypertrophy are conspicuous, and there is often much atheroma. The intimal thickening is sometimes of extreme degree.

The Heart and other Organs. In the heart, the fine fibrosis suspected macroscopically is not confirmed by histological examination but the muscle fibres of the left ventricle show rather wide separation. Chronic venous congestion and a subcapsular, probably adenomatous, nodule are present in the section of the liver; infarction and a cortical adenoma in the kidney. The blood vessels of all sizes in the heart, liver, and kidney are normal.

\section{Discussion}

Clinically the most striking features about this case were the steadily progressive symptoms and signs of right ventricular failure, unrelieved by rest in bed and full digitilization. Breathlessness on exertion was first noticed less than four months before death and there was not the slightest indication of lung or heart disease before this. During the last four weeks, although cyanosis and breathlessness on movement steadily increased, there was a notable absence of quickening of the pulse rate and respiratory rate at rest and there was practically no oedema.

In an analysis of 16 somewhat similar cases, Brenner (1935) states that the duration of symptoms varied from five months to five years, the average being less than two years, and that the course was steadily downhill, treatment being ineffective. In Navasquez' three cases (1940) the duration of symptoms in one was a year, in another two years and three months, and the third had been "chesty and bronchial" for a few years. In East's three cases (1940) it was two to three years in one, six years in another, and six months in the third. In Armstrong's (1940) it was four years. By comparison with these this case appears to represent an acute manifestation of the syndrome. It is possible that the stirring up of sepsis caused by dental extraction was an additional factor in precipitating failure.

On pathological grounds too there seems no doubt that this patient died of heart failure, and the hypertrophy and dilatation of the chambers of the right side of the heart together with the absence of any abnormality of the left side at once suggests increased resistance to the flow of blood through the lungs as the probable cause. The morbid changes in the lungs lend direct support to this view, for not only is there much disintegration of the alveolar walls with inevitable reduction of the capillary bed, but there is also widespread and severe disease of the pulmonary blood vessels. Both of these abnormalities would increase the resistance in the pulmonary circuit.

What is the relationship between the vascular lesions and the disintegration of the alveolar walls? The latter might cause vascular lesions by raising the pulmonary blood pressure, but on the other hand circulatory impairment due to vascular lesions might conceivably cause disintegration of the alveolar walls. It seemed to us that a comparison of the character of the vascular lesions with those known to be produced in the systemic circulation by hypertension might be helpful in deciding which were primary. As a result of such a comparison we are of the opinion that all of the vascular lesions are such as might reasonably be produced by hypertension alone, and that when compared with those seen in most patients who have died of emphysema or other disorders likely to have produced pulmonary hypertension they are of very unusual severity.

The occurrence of vascular changes of unusual severity in certain cases of systemic 
hypertension is now generally recognized, such patients being said to be suffering from malignant hypertension. In a study of the structural changes in the lungs in mitral stenosis, Barker and Weiss (1936) have presented evidence of an analogous condition in the pulmonary circuit- "pulmonary hypertension with malignant sclerosis"-in which the arterioles in the lungs show hyperplastic arteriolosclerosis and, rarely, necrotising arteriolitis quite similar to the lesions seen in the systemic circulation in malignant hypertension. Though we have not found acute necrotic lesions in the blood vessels of the lungs of our patient, the resemblance of the arteriolar lesions to the hyperplastic arteriosclerosis has already been noted. It seems possible that both on clinical and pathological grounds this is a case of malignant pulmonary hypertension; that a rise of pulmonary blood pressure initiated the malignant sclerosis of the pulmonary arterioles which then exaggerated the pulmonary hypertension and set up a vicious circle. A vicious circle of this kind is probably the essential feature of malignant as distinct from benign hypertension and it explains the rapid worsening and early death of the patient.

If the vascular lesions were the result of pulmonary hypertension it is necessary to enquire how such hypertension arose. There is no satisfactory answer to this question. Undoubtedly the capillary bed was considerably reduced by disintegration of the alveolar walls, but it is impossible to do more than speculate as to whether this was sufficient to account for the hypertension and the origin of the alveolar disintegration remains a mystery. 'The most obvious explanation, that the patient was suffering from chronic emphysema, appears to us untenable. There was no clinical evidence of emphysema; no long history of cough or other chest trouble and none of the physical signs of emphysema, while post-mortem the existence of emphysema was not at first noticed, so slight was it. It cannot be denied that emphysema was present in the anterior borders of the lungs but this emphysema was atrophic in type, being merely part of the general disintegration of the lung, and there was no distension of the lung nor any formation of bullae. Thus the condition of the lungs was quite different from that seen in typical chronic emphysema. Furthermore Parkinson and Hoyle (1937) have shown that any gross degree of right ventricular enlargement is uncommon even in severe cases of chronic hypertrophic emphysema. In their series of 80 cases enlargement could only be demonstrated radiologically in 18 and in only 4 of these was it great. The case we are describing showed no clinical emphysema.

\section{SUMMARY}

- A patient is described who died of right ventricular failure within four months of the first appearance of symptoms. There was no previous history of heart, lung, or other disease. Progress was steadily downhill without any response to treatment.

Post-mortem, there was macroscopically conspicuous atheroma of the main pulmonary arteries and all its larger branches with great dilatation of the right side of the heart and hypertrophy of the right ventricle, the left side of the heart being normal. - Emphysema was only visible along the anterior borders of the lungs and there was no distension of the lungs or formation of bullae.

Microscopically, there was conspicuous medial and intimal hypertrophy in the larger pulmonary arteries with much atheroma, and great intimal thickening in many of the small muscular arteries. Changes similar to hyperplastic arteriosclerosis of malignant hypertension were seen in the pulmonary arterioles. There was widespread disintegration of the alveolar walls without hypertrophic emphysema.

The significance of these changes is discussed.

We wish to thank Dr. Ff. Roberts for the X-ray examination, and Mr. H. P. Hudson for the photomicrographs.

\section{REFERENCES}

Armstrong, T. G. (1940). Brit. Heart J., 2, 201.

Brenner, O. (1935). Arch. intern. Med., 56, 211, 457, 724, 976, 1189.

East, T. (1940). Brit. Heart J., 2, 189.

Navasquez, S. de, Forbes, J. R., and Holling, H. E. (1940). Brit. Heart J., 2, 177.

Parker, F. and Weiss, S. (1936). Amer. J. Path., 12, 573.

Parkinson, J. and Hoyle, C. (1937). Quart. J. Med. New Series, 6, 59. 